
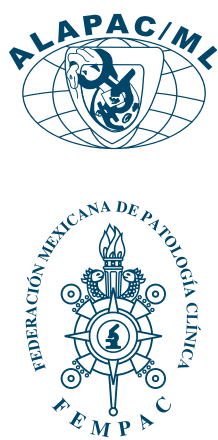

Artículo original

\title{
Concentración de 25(0H)-vitamina D en población pediátrica
}

\section{Concentration of 25(OH)-vitamin D in pediatric population}

\author{
Parra Ortega Israel, ${ }^{*,}$ Reyes Pérez Herlinda,* Domínguez Castillo Verónica, ${ }^{\S}$ \\ Espinosa Arreola Maritza, ${ }^{\ddagger}$ Olmedo Gutiérrez Eduardo, ${ }^{\ddagger}$ López Martínez Briceida ${ }^{\ddagger}$
}

Palabras clave: Vitamina D, niños, adolescentes, insuficiencia, deficiencia.

Keywords:

Vitamin D, children, adolescents, insufficiency, deficiency.

* Departamento de Laboratorio Clínico, Hospital Infantil de México «Federico Gómez». México.

${ }^{\ddagger}$ Laboratorios Ruiz

SYNLAB. México.

$\S$ Facultad de Estudios

Superiores Zaragoza

de la Universidad

Nacional Autónoma

de México. México.

Correspondencia:

Dra. Briceida López

Martínez

Directora de Enseñanza e Investigación,

Laboratorios Ruiz

SYNLAB, México.

Blvd. Díaz Ordaz Núm.

808, Edificio C,

Fracc. Anzures, 72530,

Puebla, Puebla.

Tel: 22 2243-8100,

Ext. 3218

E-mail: briceida.lopez@

laboratoriosruiz.com

Recibido: 26/11/2021

Aceptado: 21/01/2022

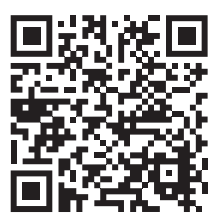

\section{RESUMEN}

Introducción: La vitamina D es fundamental en el organismo por su papel en la mineralización ósea y sus funciones extraesqueléticas. Un nivel apropiado es importante en la edad pediátrica, no obstante, la evidencia ha reportado cantidades bajas en esta población. Objetivo: Identificar los valores de vitamina $D$ en una población pediátrica que acude al Hospital Infantil de México. Material y métodos: Se realizó un estudio transversal, donde se incluyeron 222 pacientes pediátricos con medición de 25-hidroxivitamina D. Se obtuvo el porcentaje de suficiencia, insuficiencia y deficiencia por sexo y edad, en adolescentes y niños. Se determinó la concentración media de vitamina $D$ en los grupos de estudio. Resultados: Se encontró una prevalencia de $20 \%$ de deficiencia y $39 \%$ de insuficiencia de vitamina D. Los niveles bajos fueron más acentuados en el sexo femenino (61\%). En los adolescentes se encontró una concentración media menor que en niños ( $26.57 \pm 10.01$ vs $30.04 \pm 11.32$ $\mathrm{ng} / \mathrm{mL}, \mathrm{p}<0.05)$. La edad de 16 a 18 años fue el rango con mayor porcentaje de niveles bajos $(71 \%)$ y menor concentración de vitamina $D(26.20 \pm 7.85 \mathrm{ng} / \mathrm{mL})$. Conclusión: Los hallazgos encontrados en este estudio sugieren que existe una elevada prevalencia de niveles inadecuados de vitamina $\mathrm{D}$ en la población pediátrica.

\section{ABSTRACT}

Introduction: Vitamin $D$ is essential in the body due to its role in bone mineralization and its extra-skeletal functions. An appropriate level is important in the pediatric age; however, evidence has reported low amounts in this population. Objective: Identify value of vitamin $D$ in a pediatric population attending the Children's Hospital of Mexico. Material and methods: A cross-sectional study was carried out, which included 222 pediatric patients with measurement of $25(\mathrm{OH})$ vitamin $D$. The percentage of sufficiency, insufficiency and deficiency was obtained by sex and age, and in adolescents and children. The concentration of vitamin $D$ was determined in the study groups. Results: A20\% prevalence of deficiency was found, and $39 \%$ of vitamin D insufficiency. Low levels were more pronounced in the female sex $(61 \%)$. A lower mean concentration was found in adolescents than in children (26.57 \pm 10.01 vs $30.04 \pm 11.32 \mathrm{ng} / \mathrm{mL}, p<0.05$ ). The age of 16 to 18 years was the range with the highest percentage of low levels $(71 \%)$ and the lowest concentration of vitamin $D(26.20 \pm 7.85 \mathrm{ng} / \mathrm{mL})$. Conclusion: The findings in this study suggested a high prevalence of inadequate levels of vitamin $D$ in the pediatric population.

\section{INTRODUCCIÓN}

L: a vitamina $\mathrm{D}$ es considerada más una $\_$hormona que una vitamina, en el sentido de que no necesariamente procede de fuentes exógenas (alimentos), sino que aproximadamente $90 \%$ de la vitamina $D$ en nuestro cuerpo puede ser producida a partir de la exposición cutánea a la radiación solar ultravioleta B (UVB). ${ }^{1,2}$ En la dieta, existen pocos alimentos que contienen naturalmente vitamina D. El colecalciferol (vitamina D3) proviene de fuentes animales y el ergocalciferol (vitamina D2) proviene de plantas. Entre sus fuentes están el pescado azul, como sardinas, arenque, atún, caballa y salmón, además de aceite de hígado de bacalao, yemas de huevo, hongos, hígado o vísceras. ${ }^{3}$

La síntesis por exposición solar ocurre cuando el precursor 7-dehidrocolesterol presente en las células epidérmicas de la piel, se convierte por medio de la radiación UVB en previtamina D,

Citar como: Parra OI, Reyes PH, Domínguez CV, Espinosa AM, Olmedo GE, López MB. Concentración de 25(OH)-vitamina D en población pediátrica. Rev Mex Patol Clin Med Lab. 2021; 68 (2): 62-67. https://dx.doi.org/10.35366/103342 
que se isomeriza a vitamina D3. Posteriormente, la vitamina D de ambos orígenes, piel y dieta, se activan; para ello, primero sufre 25-hidroxilación en el hígado a $25(\mathrm{OH}) \mathrm{D}$ (calcidiol), la principal forma circulante de vitamina $\mathrm{D}$, con una vida media de dos a tres semanas. Luego se convierte en los riñones mediante 1-alfa-hidroxilación a su forma más activa, 1,25 (OH) 2D (calcitriol), con una vida media de 4 a 6 horas. Este proceso es impulsado por la hormona paratiroidea (PTH) y otros mecanismos, como la hipofosfatemia y la acción de la hormona del crecimiento. ${ }^{4,5}$

La principal acción de la vitamina $\mathrm{D}$ es aumentar la absorción a nivel intestinal de calcio y fósforo en particular, implicados en la mineralización ósea y la adquisición de masa ósea. ${ }^{6}$ Además de estas acciones, la vitamina D ejerce otras funciones extraesqueléticas significativas, como su función en diversos mecanismos, tales como secreción y efecto de la insulina, foliculogénesis, regulación del sistema renina-angiotensina-aldosterona, control del ciclo celular y apoptosis, además de ser moduladora en la inmunidad innata y adaptativa. ${ }^{7-9}$

Mantener niveles adecuados de vitamina D es importante para llevar a cabo estas funciones en el organismo; sin embargo, en México se ha detectado en la población infantil una elevada prevalencia de deficiencia de esta vitamina. En una investigación realizada en niños de uno a 11 años con los datos obtenidos en la Encuesta Nacional de Salud y Nutrición 2012 (ENSANUT), se encontró que, a nivel nacional, la deficiencia de vitamina D era de $25.9 \%$ en los niños en edad preescolar y de $36.6 \%$ en edad escolar. ${ }^{10}$ De igual manera, un estudio publicado en 2016, mostró una prevalencia de deficiencia de vitamina $D$ en $24.77 \%$ de una población de preescolares y escolares en la Ciudad de México. ${ }^{11}$

La disminución de vitamina $\mathrm{D}$ en la población pediátrica está considerada como factor importante de riesgo en el desarrollo de raquitismo, donde existe una alteración de la mineralización de la matriz ósea, y provoca arqueamiento de piernas, cambios en la columna vertebral como cifosis o escoliosis, baja estatura, mayor riesgo a fracturas, e incluso tetania raquítica por la hipocalcemia. ${ }^{12}$ Existen estudios que han relacionado niveles adecuados de vitamina $\mathrm{D}$ como factor protector en patologías como diabetes tipo 1, infecciones del tracto respiratorio, asma e incluso en enfermedades cardiovasculares. ${ }^{13}$

Se han descrito diversos factores asociados con la insuficiencia y deficiencia de vitamina D en población pediátrica, los cuales son desnutrición, piel oscura, obesidad, exposición solar inadecuada y patologías como enfermedad renal crónica, además de enfermedades donde existe una mala absorción intestinal, tal como en la enfermedad celiaca y enfermedad de Crohn. ${ }^{14-16}$

\section{MATERIAL Y MÉTODOS}

Diseño de estudio y selección de la muestra. Durante el periodo comprendido del 07 de mayo de 2019 al 31 de enero del 2020, se realizó un estudio observacional, transversal y descriptivo en el Hospital Infantil de México «Federico Gómez», en el que se incluyeron 222 pacientes pediátricos con medición de niveles de vitamina $\mathrm{D}$ $(25[\mathrm{OH}]$-vitamina D). La edad de los individuos fue de dos meses a 18 años, la media de esta edad fue de $9 \pm$ 5 años. En cuanto al sexo, 110 pertenecían al masculino y 112 al femenino.

Medición de vitamina D. La muestra empleada para la cuantificación de vitamina $\mathrm{D}$ fue sangre venosa, obtenida después de un ayuno de 8 horas, a partir de una muestra de suero se cuantificó la 25-hidroxivitamina D por quimioluminiscencia en la plataforma Architect i1000, de Abbott Diagnostics, siguiendo las recomendaciones del fabricante y posterior a la verificación analítica del equipo.

El analito considerado para la determinación del nivel de vitamina $\mathrm{D}$ fue $25-(\mathrm{OH})$-vitamina $\mathrm{D}$, debido a que es el mejor indicador del estado de la vitamina $D$, ya que refleja tanto producción de vitamina D3 y vitamina D dietética (D2 y D3). Además, tiene una vida media de tres a cuatro semanas. A diferencia de $25-(\mathrm{OH})-2$ vitamina D que tiene una vida media circulante corta de 4 horas y está mil veces menos en concentración que 25-(OH)vitamina D. ${ }^{17,18}$

Para la definición de suficiencia, insuficiencia y deficiencia de vitamina $\mathrm{D}$, se emplearon los valores de $<20$ $\mathrm{ng} / \mathrm{mL}$ (50 nmol/L) como deficiencia, de 20 a $30 \mathrm{ng} / \mathrm{mL}$

\begin{tabular}{|c|c|c|c|c|c|}
\hline \multirow[b]{3}{*}{ Variable } & \multicolumn{4}{|c|}{$\begin{array}{l}\text { Tabla 1: Frecuencias y porcentajes de } \\
\text { niveles inadecuados de vitamina D. }\end{array}$} & \multirow[b]{3}{*}{$p^{*}$} \\
\hline & & \multirow[b]{2}{*}{$\mathrm{N}$} & \multicolumn{2}{|c|}{ Frecuencia } & \\
\hline & & & $n$ & $\%$ & \\
\hline \multirow[t]{2}{*}{ Sexo } & Femenino & 112 & 69 & 61 & 0.538 \\
\hline & Masculino & 110 & 61 & 55 & \\
\hline \multirow[t]{2}{*}{ Etapa } & Niños & 147 & 79 & 54 & 0.120 \\
\hline & Adolescentes & 75 & 51 & 68 & \\
\hline Edad & 0 a 3 & 34 & 22 & 65 & 0.654 \\
\hline \multirow[t]{5}{*}{ (años) } & 4 a 6 & 38 & 20 & 53 & \\
\hline & 7 a 9 & 57 & 29 & 51 & \\
\hline & 10 a 12 & 27 & 14 & 52 & \\
\hline & 13 a 15 & 45 & 30 & 67 & \\
\hline & 16 a 18 & 21 & 15 & 71 & \\
\hline
\end{tabular}


Tabla 2: Concentración media de vitamina D.

\begin{tabular}{llccc} 
Variable & & $n$ & Vitamina D (ng/mL) & $p^{\ddagger}$ \\
\hline Sexo & Femenino & 112 & $28.21 \pm 11.00$ & 0.366 \\
Etapa & Masculino & 110 & $29.54 \pm 11.00$ & 0.025 \\
& Niños & 147 & $30.04 \pm 11.32$ & 0.193 \\
Edad (años) & Adolescentes & 75 & $26.57 \pm 10.01$ & \\
& 0 a 3 & 34 & $29.21 \pm 11.42$ & $29.01 \pm 8.81$ \\
& 4 a 6 & 38 & $31.59 \pm 12.70$ & $28.98 \pm 10.53$ \\
& 7 a 9 & 57 & $26.22 \pm 11.17$ & \\
& 10 a 12 & 45 & $26.20 \pm 7.85$ & \\
& 13 a 15 & 21 & & \\
\hline
\end{tabular}

* IC 95\%; ₹ Prueba t de Student y ANOVA de un factor.

(50 a $<75 \mathrm{nmol} / \mathrm{L})$ como insuficiencia y $>30 \mathrm{ng} / \mathrm{mL}$ (75 $\mathrm{nmol} / \mathrm{L})$ como suficiencia. ${ }^{19}$

Análisis estadístico. Se empleó el programa estadístico SPSS versión 25 para obtener la prevalencia de deficiencia e insuficiencia en la población de estudio. De igual manera se calculó el porcentaje de suficiencia, insuficiencia y deficiencia considerando sexo y edad, así mismo, se realizó este análisis separando a la población en adolescentes y niños. Se realizó el cálculo de la media de los niveles de vitamina $\mathrm{D}$ en los grupos de estudio.

Se utilizó la prueba estadística $\chi^{2}$ para comparación de variables cualitativas y $t$ de Student para comparar medias entre grupos, ANOVA de un factor se utilizó para determinar la diferencia de más de dos grupos, de esta manera se determinó si existía diferencia significativa de los niveles de vitamina D de acuerdo al sexo, edad, y entre niños y adolescentes.

\section{RESULTADOS}

El 59\% de los individuos presentaron niveles inadecuados de vitamina D, 20\% correspondió a deficiencia y 39\% a insuficiencia. La concentración media fue $28.87 \pm 10.93$ $\mathrm{ng} / \mathrm{mL}$. En los niveles con deficiencia, la media obtenida fue de $14.33 \pm 4.37 \mathrm{ng} / \mathrm{mL}$, en insuficiencia fue de $25.47 \pm$ $3.11 \mathrm{ng} / \mathrm{mL}$ y en suficiencia se obtuvo $38.99 \pm 7.66 \mathrm{ng} / \mathrm{mL}$.

En la Tabla 1 se muestra el porcentaje de los individuos con niveles bajos de vitamina D. El sexo femenino presenta el porcentaje más alto de deficiencia con $61 \%$, así mismo, los adolescentes mostraron un porcentaje de 68\%, más alto comparado con los niños; por último, se muestra por edad, siendo el rango de 16 a 18 años con mayor prevalencia, al presentarse $71 \%$ con niveles inadecuados de vitamina $\mathrm{D}$.
La media de vitamina $\mathrm{D}$ se mostró ligeramente más disminuida en el sexo femenino que en el masculino, con una concentración de $28.21 \pm 11$, como se expone en la Tabla 2, de igual forma, se obtuvo en el grupo de adolescentes una media $26.57 \pm 10.01 \mathrm{ng} / \mathrm{mL}$, que fue menor que la mostrada en niños, esta diferencia fue estadísticamente significativa ( $p<0.05)$. Los individuos de 16 a 18 años obtuvieron una media de $26.20 \pm 7.85$, menor que los otros rangos de edad.

Se obtuvo en las mujeres una deficiencia de vitamina D en $22 \%$ de este grupo e insuficiencia en $39 \%$, estos valores fueron más elevados que en los hombres. En cuanto a los adolescentes, la deficiencia e insuficiencia fue de 24 y 44\%, respectivamente, más alta que en los niños. Se presentó una deficiencia en $29 \%$ en la edad de 13 a 15 años, y 48\% de insuficiencia en el rango de 15 a 18 años (Tabla 3).

\section{DISCUSIÓN}

La prevalencia de niveles inadecuados de vitamina D en la población pediátrica atendida en el Hospital Infantil «Federico Gómez» fue elevada (59\%). Este valor es cercano al reportado por ENSANUT en 2006, donde se mostró que en la Ciudad de México la prevalencia era de $52 \%$ en niños preescolares y escolares. ${ }^{20}$ En este estudio se determinó que la deficiencia se encontraba en $20 \%$ y la insuficiencia en $39 \%$ en la población pediátrica. Al comparar nuestros resultados con el estudio de Toussaint, no tenemos una diferencia importante, ya que el estado de deficiencia de vitamina $\mathrm{D}$ que ellos observan es de $24.7 \%$ (4.7\% más que este estudio). ${ }^{11}$

Se observó en el sexo femenino una prevalencia más alta que en el masculino (61 vs 55\%, p > 0.05), la 
concentración de vitamina $\mathrm{D}$ fue de $28.21 \pm 11 \mathrm{ng} / \mathrm{mL}$ en mujeres y $29.54 \pm 11 \mathrm{ng} / \mathrm{mL}$ en hombres, estas diferencias no fueron significativas $(p>0.05)$. En la investigación de Flores realizada en niños de uno a 11 años, la comparación por sexo evidenció que no se encontraron diferencias estadísticamente significativas en los mismos parámetros. ${ }^{10} \mathrm{El} 68 \%$ de adolescentes mostró niveles inadecuados de vitamina D, comparado con $54 \%$ en niños $(p>0.05)$. La concentración media de esta vitamina fue de $30.04 \pm 11.32 \mathrm{ng} / \mathrm{mL}$ y $26.57 \pm 10.01 \mathrm{ng} / \mathrm{mL}$, en niños y adolescentes, respectivamente, mostrando una diferencia entre ambos grupos $(p<0.05)$. Esta diferencia ha sido encontrada en estudios similares, tal como se reportó en Durá, donde los niveles vistos en adolescentes fueron menores a un grupo de escolares $(28.8 \pm 7.56 \mathrm{ng} / \mathrm{mL}$ vs $27.02 \pm 7.75 \mathrm{ng} / \mathrm{mL}, \mathrm{p}<0.05) .{ }^{21}$ Esto concuerda con la determinación por edad, siendo el rango de 16 a 18 años el que mostró una menor concentración de vitamina $\mathrm{D}$ $(26.20 \pm 7.85 \mathrm{ng} / \mathrm{mL})$ y el mayor porcentaje de niveles inadecuados (71\%).

Tomando en cuenta los niveles bajos de vitamina D en deficiencia e insuficiencia, los hallazgos indican que la deficiencia está más presente en el sexo femenino (22\%) y en los adolescentes (24\%), así como en el rango de 13 a 15 años (29\%). De igual manera, la insuficiencia de vitamina $\mathrm{D}$ fue más acentuada en mujeres (39\%) y adolescentes (44\%), y en la edad de 16 a 18 años (48\%). El porcentaje más elevado de suficiencia fue de siete a nueve años (49\%).

Esta alta prevalencia puede atribuirse a diferentes factores de riesgo a los que está expuesta la población pediátrica que asiste al Hospital Infantil de México «Federico Gómez», pues todos los pacientes que acuden a nuestro hospital cursan o tienen una enfermedad crónico-degenerativa o compleja. Una adecuada nutrición es parte importante en el aporte de vitamina D. En México, la Encuesta Nacional de Salud y Nutrición de 2012 señaló que en los niños de seis a 12 años las ingestas promedio de dicha vitamina son sumamente bajas, y que la dieta en los niños mexicanos en edad escolar por sí sola, incluyendo el consumo de leche fortificada, es insuficiente para cubrir los requerimientos diarios. ${ }^{22}$ De la misma manera, se ha detectado que en los neonatos alimentados exclusivamente con leche materna, hay una mayor predisposición a presentar deficiencia de vitamina $\mathrm{D}$, debido a que contiene cantidades muy bajas (25-78 UI/L), como se señala en el estudio de Ochoa. ${ }^{23}$

Existen factores de riesgo que dificultan que la exposición solar sintetice la vitamina $\mathrm{D}$, uno de ellos es el fototipo cutáneo, los cuales se clasifican del I al VI, según la sensibilidad de la piel frente a la luz ultravioleta. Según se incremente el número del fototipo cutáneo, la piel es más oscura, factor que ha sido relacionado con el déficit de vitamina D. ${ }^{24}$

La hipovitaminosis $\mathrm{D}$ se ha relacionado con la obesidad. En la investigación de Flores se encontró que los niños en edad escolar con sobrepeso/obesidad tenían un mayor riesgo de deficiencia de vitamina $\mathrm{D}$, en comparación con los niños de peso normal (OR $=2.23$; IC 95\% $1.36,3.66 ; p<0.05)$. El secuestro de 25-hidroxivitamina D en el tejido adiposo y las anomalías en el metabolismo de la vitamina D son mecanismos probables detrás de esta asociación. ${ }^{10}$

Es importante identificar los factores de riesgo que puedan ser modificados para poder asegurar niveles adecuados de vitamina $\mathrm{D}$, puesto que en la población pediátrica existen consecuencias en la salud que han

Tabla 3: Frecuencias y porcentajes por deficiencia, insuficiencia y suficiencia de vitamina D.

\begin{tabular}{|c|c|c|c|c|c|c|c|c|}
\hline \multirow[b]{2}{*}{ Variable } & & \multirow[b]{2}{*}{$\mathrm{N}$} & \multicolumn{2}{|c|}{ Deficiencia } & \multicolumn{2}{|c|}{ Insuficiencia } & \multicolumn{2}{|c|}{ Suficiencia } \\
\hline & & & $n$ & $\%$ & $n$ & $\%$ & $\mathrm{n}$ & $\%$ \\
\hline \multirow[t]{2}{*}{ Sexo } & Femenino & 112 & 25 & 22 & 44 & 39 & 43 & 39 \\
\hline & Masculino & 110 & 19 & 17 & 42 & 38 & 49 & 45 \\
\hline \multirow[t]{2}{*}{ Etapa } & Niños & 147 & 26 & 18 & 53 & 36 & 68 & 46 \\
\hline & Adolescentes & 75 & 18 & 24 & 33 & 44 & 24 & 32 \\
\hline \multirow[t]{6}{*}{ Edad (años) } & 0 a 3 & 34 & 6 & 18 & 16 & 47 & 12 & 35 \\
\hline & 4 a 6 & 38 & 6 & 16 & 14 & 37 & 18 & 47 \\
\hline & 7 a 9 & 57 & 10 & 18 & 19 & 33 & 28 & 49 \\
\hline & 10 a 12 & 27 & 4 & 15 & 10 & 37 & 13 & 48 \\
\hline & 13 a 15 & 45 & 13 & 29 & 17 & 38 & 15 & 33 \\
\hline & 16 a 18 & 21 & 5 & 24 & 10 & 48 & 6 & 28 \\
\hline
\end{tabular}


sido descritas en diversas investigaciones. Entre ellas está la importante asociación de hipovitaminosis D con el desarrollo de raquitismo; además, se considera que el pico de masa ósea es alcanzado en edades tempranas de la vida y éste se acepta como un predictor de riesgo de osteoporosis en la edad adulta, por lo que se deben optimizar desde la infancia los factores modificables que puedan afectar a la masa ósea, incluido el estado de la vitamina D. ${ }^{25}$

Un adecuado nivel de vitamina $\mathrm{D}$ en edad pediátrica se ha asociado a una menor prevalencia de diversas enfermedades, dado que la vitamina $\mathrm{D}$ tiene un importante rol en el sistema inmune, modulando la inmunidad innata y adaptativa. ${ }^{9}$ Entre ellas están las infecciones respiratorias, asma, dermatitis y diabetes tipo I. ${ }^{26,27}$ Incluso en estudios realizados durante la pandemia de COVID-19, se ha hallado que el estado de la vitamina $\mathrm{D}$ en la sangre puede determinar las posibilidades de contraer el virus, la gravedad de la enfermedad y la mortalidad, debido a su notable papel de la vitamina $D$ en la inmunidad. ${ }^{28,29}$

Es necesario que se evalúen las causas más probables que provocan niveles inadecuados de vitamina $D$ en la población pediátrica, ya que esto implica su asociación con diversas patologías, para que de esta manera se pueda realizar una prevención y disminuir la elevada prevalencia mostrada en este estudio.

\section{CONCLUSIÓN}

La población pediátrica estudiada tiene un nivel alto de insuficiencia y deficiencia de 25-hidroxivitamina D, considerando que las limitaciones de este estudio son los pacientes pediátricos atendidos por enfermedades de alta especialidad, por lo que es importante estudiar poblaciones clínicamente sanas.

\section{REFERENCIAS}

1. Bover J, Egido J, Fernández-Giráldez E, Praga M, Solozábal-Campos C, Torregrosa JV et al. Vitamina D, receptor de la vitamina D e importancia de su activación en el paciente con enfermedad renal crónica. Nefrología (Madr.) 2015; 35 (1): 28-41.

2. Cucalón-Arenal JM, Blay-Cortés MG, Zumeta Fustero J, Blay Cortés V. Actualización en el tratamiento con colecalciferol en la hipovitaminosis D desde atención primaria. Med Gen Fam. 2019; 8 (2): 68-78.

3. Saggese G, Vierucci F, Prodam F, Cardinale F, Cetin I, Chiappini E et al. Vitamin D in pediatric age: consensus of the Italian Pediatric Society and the Italian Society of Preventive and Social Pediatrics, jointly with the Italian Federation of Pediatricians. Ital J Pediatr. 2018; 44: 51.

4. Chang SW, Lee HC. Vitamin D and health - The missing vitamin in humans. Pediatr Neonatol. 2019; 60 (3): 237-244.
5. Navarro-Moreno MA, Alía-Ramos P. Metabolismo óseo. Vitamina D y PTH. Endocrinol Nutr. 2006; 53 (3): 199-208.

6. Hawkins-Carranza F. La vitamina D3 y el hueso. REEMO. 2007; 16 (3): 45-47.

7. Instituto Linus Pauling. [Internet] Vitamina D. [Consultado el 24 de octubre de 2020] Disponible en: https://lpi.oregonstate.edu/es/mic/ vitaminas/vitamina-D

8. García-Gil A, Sánchez-Armendáriz K, Domínguez-Cherit J. La vitamina $\mathrm{D}$ en dermatología: una revisión de la literatura. Dermatol Cosm Méd y Quir. 2013; 11(4): 270-276.

9. Mailhot G, White JH. Vitamin D and immunity in Infants and Children. Nutrients. 2020; 12 (5): 1233.

10. Flores A, Flores $M$, Macias N, Hernández-Barrera L, Rivera $M$, Contreras A et al. Vitamin D deficiency is common and is associated with overweight in Mexican children aged 1-11 years. Public Health Nutr. 2017; 20 (10): 1807-1815.

11. Toussaint-Martínez de Castro G, Guagnelli MA, Clark P, MéndezSánchez L, López-González D, Galán-Herrera JF et al. Vitamin D, not iron, is the main nutrient deficiency in pre-school and schoolaged children in Mexico City: a cross-sectional study. Nutr Hosp. 2016; 33 (4): 794-800.

12. Riancho JA. Osteomalacia y raquitismo. REEMO. 2004; 13 (4): 77-79.

13. Ashraf M, Ishaq M, Parrey NA, Irshad M, Ahmed K. Vitamin D de deficiency in children. Asian J Med Sci. 2015; 6 (1): 1-7.

14. Alonso López C, Ureta Velasco N, Pallás Alonso CR, Pallás Alonso CR. Vitamina D profiláctica. Rev Pediatr Aten Primaria. 2010; 12 (47): 495-510.

15. Cediel G, Pacheco-Acosta J, Castillo-Durán C. Deficiencia de vitamina D en la práctica clínica pediátrica. Arch Argent Pediatr. 2018; 116 (1): e75-e81.

16. Sánchez A, Oliveri B, Mansur JL, Fradinger E. Diagnóstico, prevención y tratamiento de la hipovitaminosis D. Rev Argent Endocrinol Metab. 2013; 50 (2): 140-156.

17. Reyes-Domínguez A, Gómez-De Tejada RR, Sosa-Henríquez M. La vitamina D. Fisiología. Su utilización en el tratamiento de la osteoporosis. Rev Osteoporos Metab Miner. 2017; 9 (1): 5-9.

18. Serrano Díaz N, Guío Mahecha E, González A, Plata Paredes L, Quintero Lesmes DC, Becerra Bayona S. Cuantificación de vitamina D: de la investigación a la práctica clínica. Biosalud. 2017; 16 (1): 67-79.

19. De la Calle-Cabrera T. Raquitismo carencial. Raquitismos resistentes. Pediatr Integral. 2015; 19 (7): 477-487.

20. Flores M, Sánchez LM, Macías N, Lozada A, Díaz E, Barquera $S$. Concentraciones séricas de vitamina $D$ en niños mexicanos. Cuernavaca, México: Instituto Nacional de Salud Pública; 2006.

21. Durá-Travé T, Gallinas-Victoriano F, Chueca Guindulain MJ, Berrade-Zubiri S. Deficiencia de vitamina D en escolares y adolescentes con un estado nutricional normal. Nutr Hosp. 2015; 32 (3): 1061-1066.

22. Flores ME, Macías-Morales N, Rivera-Pasquel ME. Efectos de la vitamina $\mathrm{D}$ sobre la salud, la respuesta inmune y el neurodesarrollo en niños. Revisión de la literatura. México: Instituto Nacional de Salud Pública; 2012. Disponible en: https://www.insp.mx/ resources/images/stories/Produccion/pdf/130530_efectos vitaminaD.pdf

23. Ochoa-Correa EC, García-Hernández PA, Villarreal-Pérez JZ, Treviño-Garza C, Rodríguez-Balderrama I, Martínez-de Villarreal LE et al. Deficiencia de vitamina $\mathrm{D}$ en madres y neonatos mexicanos. Gac Med Mex. 2017; 153 (5): 559-565.

24. Acuña Aguilarte PM, Jiménez Acosta SM, Muñoz Pérez JV, Esquivel Lauzurique M. Suplementación con vitamina D en las edades pediátricas. Rev Cubana Med Gen Integr. 2016; 32 (3): 1-12. 
25. Martínez-Suárez V, Moreno-Villares JM, Dalmau-Serra J. Recomendaciones de ingesta de calcio y vitamina D: posicionamiento del Comité de Nutrición de la Asociación Española de Pediatría. An Pediatr (Barc). 2012; 77 (1): 57.e1-57.e8.

26. Zanchetta MB, Fradinger E. Vitamina D. Separata Línea Montpellier. 2009; 17 (6): 1-48.

27. Cepeda SJ, Zenteno AD, Fuentes SC, Bustos BR. Vitamina D y enfermedades respiratorias pediátricas. Rev Chil Pediatr. 2019; 90 (1): 94-101.
28. Álvarez-López J, García-Contreras A. Vitamina D y la pandemia por COVID-19. Rev Mex Endocrinol Metab Nutr. 2020; 7: 95-101.

29. Liu N, Sun J, Wang X, Zhang T, Zhao M, Li H. Low vitamin D status is associated with coronavirus disease 2019 outcomes: A systematic review and meta-analysis. Int J Infect Dis. 2021; 104: 58-64.

Financiamiento: Ninguno.

Conflicto de intereses: Ninguno. 